\title{
La formation continue des directeurs comme outil managérial d'introduction du changement en gestion scolaire
}

\section{Kaoutara ELOMARI}

\author{
Université Mohammed V Souissi, Faculté des sciences de l'éducation, Rabat.
}

\section{Résumé}

L'amélioration de la gestion scolaire est devenue une nécessité dans les systèmes éducatifs vue les contraintes des changements actuels, c'est pourquoi le développement du rôle du directeur est placé en priorité et c'est ainsi qu'on mise actuellement sur la formation continue de ce dernier.

On cherche à ce que cette formation contribue à impliquer les chefs des établissements scolaires dans le processus de changement et à assurer une transition réelle chez ces acteurs pour qu'ils s'engagent volontairement et effectivement dans l'innovation de la gestion de leurs écoles.

L'expérience Marocaine montre que suite à des processus continus de réforme, les décideurs sont devenus conscients de la nécessité de s'orienter dans un sens managérial et de considérer l'innovation dans la gestion scolaire et le rôle du directeur comme point de départ pour arriver à une performance du système.

Parmi les outils exploités pour atteindre ces objectifs, la formation continue des directeurs est venue en premier lieu.

Cependant l'étude du terrain montre que cette formation comme conçue et dispensée n'a pas $\mathrm{pu}$ assurer l'engagement total des chefs des établissements scolaires dans l'introduction du changement voulu.

\section{Mots clés :}

Changement- directeurs- formation continue- gestion scolaire- transition.

\section{Introduction}

Dans tous les systèmes éducatifs la question de changement et d'innovation est devenue une réalité qui s'impose dans un contexte économique, politique et social critique.

Les enjeux majeurs du changement sont rapportés d'une part à la déconcentration et décentralisation à tous les niveaux administratifs, dont celui de l'éducation et de formation, en vue d'assurer une bonne gouvernance et d'autre part aux caractéristiques d'une nouvelle société en mutation rapide et en recherche d'ouverture de ces systèmes dans un sens de complémentarité et de développement durable.

A ces contraintes, s'ajoutent évidemment les conditions internes propres à chaque système.

L'exemple du système éducatif marocain illustre clairement cette tendance croissante de changement qui se manifeste par les processus de réformes mis en œuvre dés le début du $21^{\text {ème }}$ siècle.

Etant conscient que le changement du système éducatif doit se construire grâce au facteur humain et à l'échelle de l'école en premier lieu, on doit croire au rôle que jouent les directeurs des établissements scolaires comme partie prenante et indispensable à la conduite du changement et on doit faire intervenir donc deux composantes importantes qui constituent des besoins de base :

- L'implication du directeur et son engagement dans l'introduction du changement. 
- La formation continue du directeur comme outils de management capable d'assurer l'introduction du changement.

Dans le cadre de ce travail nous avons essayé d'étudier ces deux points dans une logique de recherche de corrélation pour répondre à la question :

\section{Comment la formation continue des directeurs a-t-elle affecté l'engagement de ces} derniers dans l'introduction du changement au niveau de la gestion scolaire?

Pour développer cette question nous allons aborder d'une part une analyse théorique dans le but de démontrer l'importance de l'implication des directeurs dans le changement et le rôle de la formation continue dans ce sens. D'autre part on testera la relation entre ces deux facteurs en présentant les résultats d'une enquête réalisée auprès d'un échantillon de directeurs d'établissements scolaires marocains.

\section{Mener le changement en gestion scolaire, un problème de transition}

Le changement est défini par (Colerette et al, 1997) comme «tout passage d'un état à un autre, qui est observé dans l'environnement et qui a un caractère durable » ${ }^{1}$

Selon (Bridge, 1995), «un changement est une modification objective de notre environnement débutant généralement par une «nouveauté ». Il vient perturber notre équilibre établi....il est extérieur à soi et daté dans le temps ${ }^{2} »$.

Ce qui caractérise le changement donc c'est qu'il apporte une modification observable ou des éléments nouveaux qui viennent changer le statuquo.

La modification est généralement factuelle émanant des promoteurs du changement qui peuvent ne pas faire partie du système en question, mais l'introduction effective du changement nécessite le passage par plusieurs phases qui forment ce qu'on peut appeler: processus du changement. Ce processus fait référence à l'expérience de ceux qui vivent et intègrent le changement.

En lien avec les théories de changement, on parle souvent de l'innovation comme moyen pour assurer le changement.

Pour (Perrenoud 2003) l'innovation est «une stratégie volontariste de certains acteurs pour favoriser voir provoquer certains changements $»^{3}$

La définition fait intervenir le critère de volonté des acteurs qui peut favoriser le changement.

Il est certain donc que les créateurs du changement doivent avoir cette volonté, mais la motivation doit émaner surtout de ceux qui subissent le changement et qui sont en principe les plus concernés et les plus capables de le faire réussir ou non.

Le problème qui se pose donc c'est la résistance au changement.

Nous avons dit que le changement est un passage d'un état d'équilibre à un état de déséquilibre dans l'attente d'un nouvel équilibre souhaité. Cette phase intermédiaire est généralement critique car en général les systèmes ont tendance à protéger leur état de stabilité et le réflexe naturel de leurs éléments sociaux c'est la résistance au changement.

\footnotetext{
${ }^{1}$ Collerette. P; Delisle. G; Perron. R, le changement organisationnel : théories et pratiques, Presse de l'université du Québec, Québec, 1997.

${ }^{2}$ Simon. L, Accompagner le changement en éducation, dans Pelletier. G, Accompagner les réformes et les innovations en éducation L'HARMATAN, 2004

${ }^{3}$ Perrenoud. F, pourquoi et comment rendre les établissements scolaires innovateurs? Bulletin de l'UNETP, n86 février 2003
} 
Il ne s'agit pas tout le temps de réactions directes d'hostilité car la résistance peut rester indirecte et le refus implicite peut avoir des effets plus dangereux pour le changement.

S'il est nécessaire de prévoir une planification du changement c'est-à-dire des phases de son introduction, il est plus important de préparer les acteurs concernés, de chercher les moyens pour assurer leur engagement et de les accompagner et parrainer durant les phases critiques. C'est ainsi qu'on peut assurer l'atteinte d'une transition réelle grâce à l'intégration positive des directeurs qui doivent avoir une pensée de changement.

La transition dont on parle est un processus qui fait référence aux mouvements de transformations intérieures auxquelles l'individu sera confronté elle prend donc le temps nécessaire pour que les acteurs parviennent à s'adapter au changement.

L'innovation s'enclenche donc lorsque l'utilisateur qui n'était pas intéressée commence à se prendre au jeu et apporte les ajustements nécessaires.

\section{Place de l'établissement scolaire et du directeur dans la réforme du système éducatif marocain.}

Avec les prérogatives de la charte nationale d'éducation et de formation, la décennie de la réforme 2000-2009 a visé comme premier objectif d'assurer la décentralisation et déconcentration du système. La principale réalisation donc était la mise en place des 16 académies régionales.

Pour répondre au besoin d'améliorer la gouvernance du système, un processus de planification stratégique et opérationnelle a été mis en place au niveau national régional et provincial avec une conscience de la nécessité du développement du système d'information.

En 2005 Le programme PROCADEM (Projet de Renforcement des Capacité Institutionnelles du Système Educatif Marocain), de durée de 5ans, a été initié en partenariat avec le CANADA visant essentiellement, dans le cadre d'instauration de la bonne gouvernance, la sensibilisation à l'utilisation du management participatif, la gestion axé sur les résultats et la planification stratégique.

En parallèle, le programme GENIE qui est la concrétisation de la stratégie nationale de généralisation des NTIC dans l'éducation a été lancé en 2005.

Et dans le cadre d'un nouveau souffle donné à la réforme, le plan d'urgence 2009-2012 s'est traduit par le programme NAJAH qui a mis la question de gestion des établissements scolaires parmi les priorités.

Malgré tous les efforts, les dysfonctionnements persistent toujours et on note l'absence de procédures de gestion formalisées favorisant l'efficacité et la cohérence des modes de fonctionnement avec une faible intégration des établissements scolaires dans les processus de planification opérationnelle.

Pour ces raisons le programme PAGESM (programme d'appui à la gestion des établissements scolaires) s'est lancé en 2012 remplaçant le projet PROCADEM.

S'étalant sur 4 ans les objectifs tracés par le PAGESM sont: Appuyer les projets d'établissements, former les directeurs d'écoles et normaliser les critères de leur sélection ${ }^{4}$.

On remarque donc que l'établissement scolaire est au cœur du changement et que les directeurs constituent actuellement un élément clé dans sa réussite.

Plusieurs conditions justifient le besoin de changement en gestion scolaire. D'une manière générale, l'établissement scolaire est une organisation complexe marquée par l'ambiguïté des

\footnotetext{
${ }^{4}$ La correspondance 2-2440 relative au plan d'exécution du projet d'appui à la gestion des établissements scolaires au Maroc (PMOP-PAGESM), 1 Octobre 2012
} 
relations causes-effets, la difficulté de la prise de décision et du consensus, l'existence de l'imprévisible et de l'incertitude... et en particulier l'établissement scolaire au Maroc souffre de plusieurs problèmes d'efficacité dus aux conditions du travail, aux problèmes des profils, de formation et d'évaluation. C'est pourquoi des signes de démotivation et d'insatisfaction professionnelle apparaissent, surtout de la part des dirigeants des établissements qui sont appelés à gérer cette situation problématique et à relever les défis déjà cité.

\section{Les besoins nouveaux en gestion scolaire}

L'ensemble des constatations précédentes à propos de l'établissement scolaire justifient le besoin de changement dans sa gestion pour accompagner l'innovation des systèmes.

Il s'agit d'un besoin transformationnels pour passer :

- D'une culture de verticalité à une culture d'horizontalité

- D'une organisation hiérarchique à un management participatif

- Du directeur expert au directeur partenaire ayant le pouvoir d'influence et non de domination.

- D'une organisation stable et monotone à une organisation réactive et ouverte.

L'innovation au sein de l'établissement scolaire peut prendre des formes différentes (autonomie de gestion, démarche qualité, projet d'établissement, conseil de gestion...)

Cette innovation institutionnelle n'est pas recherchée en soi mais c'est l'atteinte d'une transition grâce à l'intégration positive des acteurs principaux dans un processus continue de rénovation (l'aspect psychologique du changement joue un rôle essentiel dans ce sens, se sont les émotions positives ou négatives des acteurs qui déterminent la durée du processus de transition)

Les nouvelles pratiques en gestion de l'établissement scolaire montrent déjà la différence et mettent l'accent sur les facteurs essentiels de l'innovation :

- La direction systémique qui répond au besoin d'une organisation qui s'adapte aux changements de son environnement. Une approche qui traite la question d'organisation et gestion de l'établissement scolaire dans sa globalité d'une manière à assurer le passage d'une organisation stable et monotone à une organisation réactive et ouverte et d'une culture de verticalité à une culture d'horizontalité et d'interaction.

- La notion du leadership qui occupe toujours une place importante dans la recherche en management scolaire, pour parler maintenant d'un leadership transformationnel et coopératif qui répond au besoin de dévolution du pouvoir et responsabilisation du groupe de travail.

Dans le cadre d'une étude faite pour améliorer la pratique de la direction scolaire l'OCDE a relevé 4 grands moyens d'actions ${ }^{5}$ :

1. Redéfinir les responsabilités de la direction de l'établissement scolaire pour délimiter le cadre de l'autonomie de gestion attribué.

2. Répartir les fonctions de direction d'établissement

3. Acquérir les compétences nécessaires à un exercice efficace des fonctions de direction (rôle d'une formation innovante)

\footnotetext{
${ }^{5}$ Pont. B; Nusch. D; Moorman. H, Améliorer la direction des établissements scolaires Volume 1: politiques et pratiques, Editions OCDE, Paris, 2009
} 
4. Faire de la direction des établissements scolaires une profession attrayante

Si les champs d'action préconisés par l'OCDE visent un changement des pratiques de gestion, le souci d'acquérir ces compétences nouvelles est présent et donc la problématique de déterminer le profil du directeur.

Michel Tilman et Nouria Ouali, décrivent le profil général du chef de l'établissement scolaire dans le cadre d'une culture de changement et déterminent ainsi un ensemble d'attributs et des démarches générales.

Ils avancent qu'ayant une pensée de changement le chef d'établissement scolaire novateur est un insatisfait, anticipe les problèmes, est volontaire devant les événements, interprète de façon ouverte les règles administratives, déploie une pensée systémique, dispose de références théoriques et les mobilise, recherche le pouvoir à travers son implication dans l'investissement novateur...

Concernant ses qualités c'est une personne expérimenté, légitime aux yeux de ces collègues et se donne un statut qui gère la distance entre lui et ses collaborateurs.

Et comme démarches le chef d'établissement stimule mais ne porte pas les projets, met en place des équipes autonomes, utilise des relais, encourage, stimule et valorise, crée une morale du changement, sert d'interface entre les ressources externes et les besoins internes liés au projet...

La remarque qu'on peut faire c'est que ces compétences citées ne relèvent pas d'une démarche simple d'apprentissage, elle sont associées à des situations complexes qui nécessite la réunion d'un nombre d'aptitudes et qualités qui ne peuvent faire l'objet d'un acte de formation unique ou simpliste (transmission des connaissances)

La complexité de ces actes démontre donc que la formation des directeurs doit se pencher vers la maitrise du complexe.

\section{La formation continue comme moyen d'implication des directeurs dans le changement}

Le problème qui se pose c'est que les directeurs, dans nos écoles, ne disposent pas des compétences nécessaires pour assurer la gestion souhaitée et résoudre les problèmes professionnels qui s'imposent dans cette logique de changement. Ils sont en majorité des anciens enseignants qui ne disposent d'aucune formation en gestion.

Si le besoin est exprimé en termes de compétences des directeurs des établissements, c'est donc par la formation continue qu'on peut apporter la solution à cette problématique.

La formation continue demeure la solution qui peut permettre de :

- S'adapter à la rapidité du développement et au changement du rapport avec le travail.

- De rapprocher les cultures et les modes de pensé et d'agir au sein des organisations.

- D'assurer la complémentarité et l'échange des connaissances et compétences.

C'est donc cette perspective d'innovation qui constitue actuellement l'enjeu majeur du développement de la formation continue.

\footnotetext{
${ }^{6}$ Tilman. M; Ouali. N ; Piloter un établissement scolaire, DE BOECK, Bruxelle, 2001
} 
En fait la formation continue est un facteur essentiel qui répond activement au besoin d'innovation car en la considérant comme processus de transformation et comme moyen d'acquisition et développement des connaissances et compétences elle devient donc le noyau de l'innovation et de l'introduction du changement.

On doit dire donc que si on est convaincu que le changement doit se faire surtout au niveau de l'établissement scolaire et si le directeur de l'établissement scolaire est l'un des acteurs principaux qui doivent conduire le changement, c'est la formation continue qui doit répondre à ce besoin de créer une professionnalité nouvelle.

Mais si on tend à changer les paradigmes de la direction de l'établissement scolaires ceux de la formation continue doivent changer aussi.

Pour Françoise Cros, l'innovation contient en elle-même des germes de créativité liée au contexte professionnel.

«Un des aspects de la professionnalité serait celui de la créativité opérationnalisée au cœur même de la pratique professionnelle. Or il semble que cette facette de l'action soit niée dans les formations professionnelles traditionnelles. ${ }^{7}$

Une nouvelle vision de la formation continue des directeurs s'impose donc pour que le directeur croie à la légitimité et à la faisabilité du changement, apporte les ajustements nécessaires, mobilise toutes ses ressources et développe même sa propre recherche.

\section{Mesure de l'effet d'une formation continue sur l'engagement des directeurs dans l'introduction du changement en gestion scolaire}

Si on a démontré que la formation continue doit répondre à plusieurs conditions pour assurer le changement, qu'on est il donc du cas au Maroc et jusqu'à quel point la formation continue des directeurs dans notre système a-t-elle pu favoriser l'engagement de ces derniers dans le changement?

Pour répondre à cette question nous avons effectué une enquête de terrain auprès d'un échantillon de 24 établissements du secondaire qualifiant dans la région Fès-Boulmen équipés tous de salle multimédias, outils informatiques, ordinateur portable pour le directeur et connexion internet nous avons pris l'exemple de la formation continue des directeurs en technologies de l'information et de communication TIC tout en spécifiant trois domaines (l'utilisation de la bureautique, des logiciels de gestion scolaire ou systèmes de gestion des bases de données et d'internet) et enfin on a essayé d'analyser les points suivants :

- Le niveau d'utilisation des TIC par les directeurs avant et après la formation.

- Les raisons d'introduction ou non du changement en utilisation des TIC après la formation.

Le niveau d'utilisation des TIC par les directeurs avant et après la formation

Concernant la situation initiale, l'objectif était de savoir quels sont les domaines de TIC que les directeurs utilisent en gestion scolaire et d'étudier leur niveau selon ce qu'ils jugent?

La question posée dans ce sens était :

Quels sont les domaines de TIC que vous exploitez dans la gestion de votre établissement avant la FC et comment vous juger votre niveau?

Et pour évaluer la situation après la formation nous avons posé la question :

Comment est devenu votre niveau d'utilisation des TIC après la formation et quels sont les domaines nouvellement introduit?

\footnotetext{
${ }^{7}$ Cros. F, L'agir innovationnel, entre créativité et formation, De Boeck, Bruxelle, 2007
} 
On peut récapituler les résultats obtenus pour ces deux questions dans le tableau qui suit :

\begin{tabular}{|l|c|c|c|c|c|c|}
\hline & \multicolumn{2}{|l|}{ Non utilisé } & \multicolumn{2}{l|}{ Utilisation faible } & \multicolumn{2}{l|}{ Utilisation avancée } \\
\hline & avant & après & avant & après & avant & après \\
\hline bureautique & $12,5 \%$ & $8,5 \%$ & $33,5 \%$ & $29 \%$ & $54 \%$ & $62,5 \%$ \\
\hline $\begin{array}{l}\text { SGBD et logiciels } \\
\text { de gestion scolaire }\end{array}$ & $92 \%$ & $70,5 \%$ & $8 \%$ & $12,5 \%$ & $0 \%$ & $17 \%$ \\
\hline Internet & $4 \%$ & $0 \%$ & $83,5 \%$ & $62,5 \%$ & $12,5 \%$ & $37,5 \%$ \\
\hline
\end{tabular}

Le tableau montre qu'actuellement pour les directeurs enquêtés, il y a un grand taux d'utilisation avancée de la bureautique $62,5 \%$, un grand nombre n'utilise pas les logiciels de gestion et les SGBD, et l'utilisation d'internet est faible.

Avec un test de Khi 2 on a comparé les fréquences obtenues pour voir est ce qu'il y a une différence entre la situation des répondants avant et après la formation et les résultats on démontré que les différences observés ne sont statistiquement significatifs que pour la composante internet.

La formation en internet a eu un effet positif même si ce dernier est encore faiblement utilisé.

Des suggestions peuvent se mettre donc que le changement réalisé en cette composante seulement est lié à la motivation personnelle des directeur pour ce domaine très attrayant ou à leur propre conviction de son importance dans la vie professionnelle et personnelle aussi.

Pour trouver des explications au comportement des directeurs nous avons essayé donc de rechercher les raisons de non introduction du changement en utilisation des TIC après la formation.

Les raisons de non introduction du changement en utilisation des TIC après la formation.

La question posée est la suivante:

Si vous n'avez pas introduit ou amélioré l'utilisation de certains domaines de TIC dans votre établissement après la formation quelles sont les raisons?

Nous avons proposé des suggestions aux répondants pour les classer dans une échelle de Lickert de 1= pas du tout d'accord à 5= tout à fait d'accord :

- Je ne vois pas l'utilité de ces domaines dans la gestion de mon établissement.

- La formation ne répond pas aux besoins du terrain.

- La formation n'était pas suffisante.

- L'application de la formation sur le terrain pose une grande difficulté.

Les résultats obtenus sont comme suit :

\begin{tabular}{|c|c|c|c|c|}
\hline & minimum & maximum & moyenne & Ecart-type \\
\hline Je ne vois pas l'utilité de ces domaines dans mon travail & 2 & 5 & 4,1 & 0,9 \\
\hline La formation ne répond pas aux besoins du terrain. & 1 & 5 & 3,6 & 1,2 \\
\hline La formation n'était pas suffisante. & 1 & 5 & 3,1 & 1,5 \\
\hline L'application sur le terrain pose une grande difficulté. & 3 & 5 & 4,5 & 0,6 \\
\hline Nombre des répondants & \multicolumn{4}{|c|}{1} \\
\hline
\end{tabular}


La première observation est que les quatre propositions présentent aussi un score élevé (une moyenne supérieur à 3)

La conclusion principale qu'on peut retirer, en analysant les moyennes pour chaque item, c'est que les réponses s'orientent plus vers deux explications :

1. L'application de la formation sur le terrain pose une grande difficulté (avec une moyenne de 4,5)

2. Je ne vois pas l'utilité de ces domaines dans mon travail (avec une moyenne de 4,1)

L'analyse de ces deux réponses montre que le manque d'engagement dans le changement est dû surtout à deux faits distincts :

Soit que la formation continue, malgré son importance, n'a pas pu donner aux directeurs les outils convenables pour faire face au terrain marqué par la complexité.

Soit que, et c'est le plus décevant, cette formation n'a pas pu convaincre les directeurs de son utilité pour qu'ils puissent changer leurs pratiques professionnels.

Dans les deux cas il est impossible que les directeurs introduisent le changement souhaité et cela à cause de l'absence de motivation pour quelque chose qui parait soit non utile ou difficile.

\section{Conclusion}

En guise de conclusion on peut dire que dans nos établissements on a un besoin de transition plus que de changement.

L'innovation institutionnelle menée n'est pas recherchée en soi car ce qui manque c'est l'atteinte d'une transition réelle grâce à l'intégration positive des directeurs qui doivent avoir une pensée de changement.

La formation continue des directeurs des établissements scolaires doit s'orienter donc vers un accompagnement professionnel de ces derniers pour apporter des réponses aux problèmes du métier et réaliser la transition voulue. 